\title{
Resolving Triple Systems into Regular Configurations
}

\author{
E. Mendelsohn \\ Department of Mathematics, University of Toronto \\ Toronto, ON M5S 3G3 CANADA \\ mendelso@math.utoronto.ca \\ G. Quattrocchi \\ Dipartimento di Matematica, Universita' di Catania \\ Catania, ITALIA \\ quattrocchi@dipmat.unict.it
}

Submitted: June 25, 1999; Accepted: November 22, 1999

\begin{abstract}
A $\lambda-$ Triple $\operatorname{System}(v)$, or a $\lambda-T S(V, \mathcal{B})$, is a pair $(\mathrm{V}, \mathcal{B})$ where $\mathrm{V}$ is a set and $\mathcal{B}$ is a subset of the 3 -subsets of $\mathrm{V}$ so that every pair is in exactly $\lambda$ elements of $\mathcal{B}$. A regular configuration on p points with regularity $\rho$ on $l$ blocks is a pair $(\mathrm{P}, \mathcal{L})$ where $\mathcal{L}$ is a collection of 3 -subsets of a (usually small) set $\mathrm{P}$ so that every $\mathrm{p}$ in $\mathrm{P}$ is in exactly $\rho$ elements of $\mathcal{L}$, and $|\mathcal{L}|=l$. The Pasch configuration $(\{0,1,2,3,4,5\},\{012,035,245,134\})$ has $\mathrm{p}=6, l=4$, and $\rho=2$. A $\lambda-T S(V, \mathcal{B})$, is resolvable into a regular configuration $\mathbb{C}=(\mathrm{P}, \mathcal{L})$, or $\mathbb{C}-$ resolvable, if $\mathcal{B}$ can be partitioned into sets $\Pi_{i}$ so that for each $\mathrm{i},\left(\mathrm{V}, \Pi_{i}\right)$ is isomorphic to a set of vertex disjoint copies of $(\mathrm{P}, \mathcal{L})$. If the configuration is a single block on three points this corresponds to ordinary resolvability of a Triple System.

In this paper we examine all regular configurations $\mathbb{C}$ on 6 or fewer blocks and construct $\mathbb{C}$-resolvable $\lambda$-Triple Systems of order $\mathrm{v}$ for many values of $\mathrm{v}$ and $\lambda$. These conditions are also sufficient for each $\mathbb{C}$ having 4 blocks or fewer. For example for the Pasch configuration $\lambda \equiv 0(\bmod 4)$ and $v \equiv 0(\bmod 6)$ are necessary and sufficient.

$M R S C \# 05 B 07$
\end{abstract}

\section{Introduction}

The study of the way in which small configurations are germane to analysing the structure of combinatorial objects has progressed from the study of finite geometries 
[7] (for example Desargues and Pappus configurations) to using small configurations in the analysis of other designs. The concepts of avoidance of $[1,13]$, ubiquity of [16], decomposability into[10], and bases for[9],small configurations, have all provided insights into the structure of designs.

On the other hand resolvability and $\lambda$-resolvability have had a similar but much longer history starting from Euclid's fifth postulate to through the end of the Euler conjecture and to the present.[6]

In this work we shall combine the two ideas into the concept of $\mathbb{C}$-Resolvable triple systems. We start with the following basic definitions:

Definition 1.1 A $\lambda$-Triple System $(v)$, a $\lambda-T S(V, \mathcal{B})$, is a pair $(V, \mathcal{B})$ where $V$ is a $v$-set and $\mathcal{B}$ is a subset of the 3-subsets of $V$ so that every pair is in exactly $\lambda$ elements of $\mathcal{B}$.

Definition 1.2 A regular configuration on $p$ points with regularity $\rho$ on $b$ blocks is a pair $(P, \mathcal{L})$ where $\mathcal{L}$ is a collection of 3 -subsets of a (usually small) set $P$ so that every $p$ in $P$ is in exactly $\rho$ elements of $\mathcal{L}$, and $|\mathcal{L}|=l$.

The Pasch configuration $(\{0,1,2,3,4,5\},\{012,035,125,134\})$ has $\mathrm{p}=6, l=4$, and $\rho=2$.

Definition $1.3 A \mathbb{C}$-parallel (or resolution) class of size $v=p t$ is a set of $v$ points together with a collection of lt lines which is isomorphic to t vertex disjoint copies of $\mathbb{C}$

Definition $1.4 A \lambda$-TS $(V, \mathcal{B})$, is resolvable into a regular configuration $\mathbb{C}=(P, \mathcal{L})$ if $\mathcal{B}$ can be partitioned into sets $\Pi_{i}$ parallel classes $i=1,2, \cdots \frac{b}{l t}$, or more simply, a triple system is called $\mathbb{C}$-resolvable iff its blocks can be partitioned into disjoint $\mathbb{C}$-parallel classes.

If the configuration is a single block on three points this corresponds to ordinary resolvability of a triple system. On the other hand if $\mathbb{C}$ is itself a $\lambda-T S(k)$, the existence of $\mathbb{C}$-resolvable resolvable $\lambda \times \mu-T S(v)$ is equivalent to the existence of a resolvable balanced incomplete block design $\operatorname{RBIBD}(v, k, \mu)$. This frames the existence problem for $\mathbb{C}$-resolvable triple systems between the concept of resolvable triple systems and resolvable block designs of other block sizes. Since not much is known about resolvable block designs with $k \geq 7$ perhaps the intermediate problem of $\mathbb{C}$-resolvable triple systems with a small number of lines will shed some light on the general problem.

We shall use $\mathbb{C}$ for a configuration with $p$ for the number of points and $l$ for the number of lines and regularity $\rho$. Further we define $\lambda_{\max }$ to be the maximal number of lines that any pair occurs in. Similarly $r e p_{\max }$ will denote the maximal number of times a block is repeated.

Lemma 1.1 The necessary conditions for a $\lambda-T S(v)$ to be $\mathbb{C}$-resolvable are 
1. $v \equiv 0(\bmod p)$

2. $\lambda(v-1) \equiv 0(\bmod 2)$

3. $\lambda \geq \lambda_{\max }$

4. Let $v=t p$ then $\lambda p(p t-1) \equiv 0(\bmod 6 l)$

5. If $\mathbb{C}=(P, \mathcal{L})$ where $\mathcal{L}$ consists of $m$ copies of the set $\mathcal{L}$ ' then necessary (and sufficient) conditions for $\mathbb{C}$ are those of $\mathbb{C}$ ' with " $\lambda$ " replaced by " $m \lambda$ "

Proof: $1,2,3$ and 5 are trivial. The number of blocks in the $\lambda-T S(v)$ is $\frac{\lambda p t(p t-1)}{6}$ which must be divisible by the number of blocks in a parallel class which is $t l$.

The solutions to the equation

$$
3 l=p \rho
$$

will be useful in classifying the regular configurations.

\section{$2 \quad \mathbb{C}-$ Resolvable Group Divisible Designs}

In order to construct the desired triple systems we shall need two auxiliary concepts. We recall the standard definition of a $k-G D D_{\lambda}(g, n)$.

Definition 2.1 A $k-G D D_{\lambda}(g, n)$ is a set $V$ partitioned into $n$, g-sets $G_{i}$ called groups together with a collection $\mathcal{B}$ of $k$-subsets called blocks so that

1. every 2-subset (pair) of elements of $V$ which are from different groups are a subset of exactly $\lambda$ blocks

2. and no block contains two elements from the same group.

Definition 2.2 A resolvable $k-G D D_{\lambda}(g, n)$ is a $k-G D D_{\lambda}(g, n)$ where $\mathcal{B}$ can be partitioned into parallel classes i.e each class contains every point exactly once.

Definition 2.3 $A k-G D D_{\lambda}(g, n)$ is $\mathbb{C}$-resolvable when $\mathcal{B}$ can be partitioned into $\mathbb{C}$-parallel classes. 
For this paper, we shall always have $k=3$ and may omit it from the notation; we may also omit $\lambda$ when $\lambda=1$.

The constructions will be based on the following variants of Wilson's Theorem.

Theorem 2.1 (Master by Ingredient) Let $\left(V_{M}, \mathcal{B}_{M}\right)$ be a resolvable 3-GDD $D_{\lambda}(g, n)$, (called the master) and $\left(V_{I}, \mathcal{B}_{I}\right)$ be a $\mathbb{C}$-resolvable

3-GDD $D_{\mu}(h, 3)$ (called the ingredient) then there exists a $\mathbb{C}$-resolvable 3-GDD $D_{\lambda \times \mu}(g h, n)$.

Theorem 2.2 (Filling in groups) Let $(V, \mathcal{B})$ be a $\mathbb{C}$-resolvable

$3-G D D_{\lambda}(g, n)$ and $\left(D, \mathcal{B}_{D}\right)$ be $\mathbb{C}$-resolvable $\lambda-T S$ with $|D|=g$. Then there exists a $\mathbb{C}-$ resolvable $\lambda-T S($ gn $)$ there exists.

The proofs of the above theorems are routine exercises based on the proofs of the original theorems found in the introductory chapter of [8].

Sometimes we have the fortuitous situation of what we shall call an $\mu$-auto ingredient configuration. That is a situation where the configuration $\mathbb{C}=(P, \mathcal{L})$ is a $\mathbb{C}$-parallel class of a $\mathbb{C}$-resolvable $3-G D D_{\mu}(g, 3), 3 g=|P|$. We give 3 examples:

Example 2.1 The trivial examples of the r-repeated block

$$
\mathbb{C}=(\{1,2,3\}, \underbrace{\{123,123 \cdots 123\}}_{\mathrm{r} \text { times }})
$$

is a $\mathbb{C}$-resolvable 3-GDD $(1,3)$.

Example 2.2 $\mathbb{C}_{4.6 .2}$ or Pasch

$$
P=\{1,2,3,4,5,6\} \text { and } \mathcal{L}=\{125,146,326,345\}
$$

This is also a $\mathbb{C}$-resolvable $3-G D D_{1}(2,3)$ with groups $\{1,3\},\{2,4\},\{5,6\}$.

Example $2.3 \mathbb{C}_{4.6 .3}$ or FIFA

$$
P=\{1,2,3,4,5,6\} \text { and } \mathcal{L}=\{125,126,346,345\}
$$

This forms one $\mathbb{C}$-resolvable class of $3-G D D_{2}(2,3)$ with groups $\{1,3\},\{2,4\},\{5,6\}$. The other is $\{145,146,236,235\}$.

Corollary 2.1 If $\mathbb{C}$ is an $\mu$ auto-ingredient configuration $(P, \mathcal{L})$ with $|P|=3 g$ and there exist a resolvable $\lambda-T S(w)$ and a $\mathbb{C}$-resolvable $\mu-T S(3 g)$, then there exists a $\mathbb{C}-$ resolvable $\lambda \times \mu-T S(g w)$. 
THE ELECTRONiC JOURNAL OF COMBinatorics 7 (2000), \#R2

\section{The regular configurations on 6 or fewer lines}

\subsection{Enumeration and Necessity}

We shall now enumerate all regular configurations on six or fewer lines and give necessary conditions for the existence of a $\mathbb{C}$-resolvable $\lambda-T S(v)$.

We shall number the configurations by $\mathbb{C}_{l . p . n}$, where $l$ is the number of lines, $p$ the number of points, and $n$ the number of the configuration.

Lemma 3.1 The enumeration of the regular configurations with $l \leq 3$ lines is as follows

The case $l=1$

$\mathbb{C}_{1.3 .1} P=\{1,2,3\}$ and $\mathcal{L}=\{123\}$.

$A \mathbb{C}_{1.3 .1}$-resolvable $\lambda-T S(v)$ is just a resolvable triple system for which the necessary conditions are $v \equiv 0(\bmod 3)$ and $\lambda$ even if $v$ is even .

The case $l=\mathbf{2}$. In this case there are two configurations

$\mathbb{C}_{2.3 .1} P=\{1,2,3\}$ and $\mathcal{L}=\{123,123\}$

$A \mathbb{C}_{2.3 .1}$-resolvable $\lambda-T S(v)$ is just a resolvable triple system with every block repeated. The necessary conditions are $v \equiv 0(\bmod 3)$ and $\lambda \equiv 0(\bmod 2)$ if $v$ is odd and $\lambda \equiv 0(\bmod 4)$ if $v$ is even.

$\mathbb{C}_{2.6 .1} P=\{1,2,3,4,5,6\}$ and $\mathcal{L}=\{123,456\}$

$A \mathbb{C}_{2.6 .1}$-resolvable $\lambda-T S(v)$ is just a resolvable triple system with an even number of blocks and the necessary conditions are $v \equiv 0(\bmod 6)$ and $\lambda \equiv 0$ $(\bmod 2)$.

The case $l=3$

$\mathbb{C}_{3.3 .1} P=\{1,2,3\}$ and $\mathcal{L}=\{123,123,123\} \quad A \mathbb{C}_{3.3 .1}$-resolvable $\lambda-T S(v)$ is just a resolvable triple system with every block repeated 3 times. The necessary conditions are $v \equiv 0(\bmod 3)$ and $\lambda \equiv 0(\bmod 3)$ if $v$ is odd and $\lambda \equiv 0$ $(\bmod 6)$ if $v$ is even.

$\mathbb{C}_{3.9 .1} P=\{1,2,3,4,5,6,7,8,9\}$ and $\mathcal{L}=\{123,456,789\}$

$A \mathbb{C}_{3.9 .1}$-resolvable $\lambda-T S(v)$ is just a resolvable triple system whose number of blocks is divisible by 3. The necessary conditions are $v \equiv 0(\bmod 9)$.

Lemma 3.2 There are six regular configurations with four lines and the necessary conditions for the existence of a $\mathbb{C}_{4 . x}$-resolvable $\lambda-T S(v)$, say $\mathcal{B}_{4 . x}$, are as follows: 
THE ELECTRONiC JOURNAL OF COMBinatorics 7 (2000), \#R2

$\mathbb{C}_{4.3 .1}$

$$
P=\{1,2,3\} \text { and } \mathcal{L}=\{123,123,123,123\}
$$

$v \equiv 0(\bmod 3)$ and $\lambda \equiv 0(\bmod 4)$ if $v$ odd, $\lambda \equiv 0(\bmod 8)$ if $v$ even.

$\mathbb{C}_{4.4 .1}$ or $2 \mathbb{K}_{4}$

$$
P=\{1,2,3,4\} \text { and } \mathcal{L}=\{123,234,341,412\}
$$

$v \equiv 4(\bmod 12), \lambda \equiv 2,4(\bmod 6)$ and $v \equiv 0(\bmod 4), \lambda \equiv 0(\bmod 6)$

$\mathbb{C}_{4.6 .1}$

$$
P=\{1,2,3,4,5,6\} \text { and } \mathcal{L}=\{123,123,456,456\}
$$

$$
v \equiv 0(\bmod 6), \lambda \equiv 0(\bmod 4)
$$

$\mathbb{C}_{4.6 .2}$ or Pasch

$$
\begin{aligned}
P & =\{1,2,3,4,5,6\} \text { and } \mathcal{L}=\{125,146,326,345\} \\
v \equiv 0(\bmod 6), \lambda & \equiv 0(\bmod 4)
\end{aligned}
$$

$\mathbb{C}_{4.6 .3}$ or FIFA

$$
P=\{1,2,3,4,5,6\} \text { and } \mathcal{L}=\{125,126,346,345\}
$$

$$
v \equiv 0(\bmod 6), \lambda \equiv 0(\bmod 4)
$$

$\mathbb{C}_{4.12 .1}$

$$
\begin{aligned}
& P=\{1,2,3,4,5,6,7,8,9, A, B, C\} \text { and } \mathcal{L}=\{123,456,789, A B C\} \\
& v \equiv 0(\bmod 12), \lambda \equiv 0(\bmod 2)
\end{aligned}
$$

Lemma 3.3 There are four regular configurations with five lines and the necessary conditions for the existence of a $\mathbb{C}_{5 . x}$-resolvable $\lambda-T S(v)$, say $\mathcal{B}_{5 . x}$, are as follows:

$\mathbb{C}_{5.3 .1}$

$$
\begin{gathered}
P=\{1,2,3\} \text { and } \mathcal{L}=\{123,123,123,123,123\} \\
v \equiv 0(\bmod 6), \lambda \equiv 0(\bmod 10) \text { and } v \equiv 3(\bmod 6), \lambda \equiv 0(\bmod 5)
\end{gathered}
$$


$\mathbb{C}_{5.5 .1}$

$$
\begin{aligned}
& \qquad P=\{1,2,3,4,5\} \text { and } \mathcal{L}=\{123,123,145,245,345\} \\
& v \equiv 0(\bmod 5), \lambda \equiv 0(\bmod 6) ; \\
& v \equiv 10(\bmod 15), \lambda \equiv 2,4(\bmod 6), \lambda \geq 3 \\
& v \equiv 5(\bmod 10), \lambda \equiv 3(\bmod 6) ; \\
& v \equiv 10(\bmod 15), \lambda \equiv 1,5(\bmod 6), \lambda \geq 3
\end{aligned}
$$

$\mathbb{C}_{5.5 .2}$

$$
P=\{1,2,3,4,5\} \text { and } \mathcal{L}=\{123,124,135,245,345\}
$$

$v \equiv 0(\bmod 5), \lambda \equiv 0(\bmod 6) ; \quad v \equiv 10(\bmod 15), \lambda \equiv 2,4(\bmod 6) ; v \equiv 5$ $(\bmod 10), \lambda \equiv 3(\bmod 6)$

$v \equiv 10(\bmod 15), \lambda \equiv 1,5(\bmod 6), \lambda \geq 2$.

$\mathbb{C}_{5.15 .1}$

$$
\begin{aligned}
& \text { and } P=\{1,2,3,4,5,6,7,8,9, A, B, C, D, E, F\} \\
& \mathcal{L}=\{123,456,789, A B C, D E F\} \\
& v \equiv 15(\bmod 30), \text { any } \lambda, \text { and } v \equiv 0(\bmod 30), \lambda \equiv 0(\bmod 2)
\end{aligned}
$$

In order to distinguish the isomorphism classes for $\mathbb{C}_{6.6 . x}$ and $\mathbb{C}_{6.9 . x}$, we shall use the invariants of number of repeated blocks, number of repeated pairs and the maximal number of disjoint blocks in the configuration.

Lemma 3.4 There are 18 regular configurations with six lines and the necessary conditions for the existence of a $\mathbb{C}_{6 . x}$-resolvable $\lambda-T S(v)$, say $\mathcal{B}_{6 . x}$, are as follows:

$\mathbb{C}_{6.3 .1}$

$$
P=\{1,2,3\} \text { and } \mathcal{L}=\{123,123,123,123,123,123\}
$$

$$
v \equiv 0 \quad(\bmod 3), \lambda \equiv 0 \quad(\bmod 6)
$$

$\mathbb{C}_{6.6 .1}$

$$
\begin{gathered}
P=\{1,2,3,4,5,6\} \text { and } \mathcal{L}=\{123,123,123,456,456,456\} \\
v \equiv 0 \quad(\bmod 6), \lambda \equiv 0 \quad(\bmod 6)
\end{gathered}
$$


THE ELECTRONiC JOURNAL OF COMBinatorics 7 (2000), \#R2

$\mathbb{C}_{6.6 .2}$

$$
\begin{gathered}
P=\{1,2,3,4,5,6\} \text { and } \mathcal{L}=\{123,123,134,256,456,456\} \\
v \equiv 0 \quad(\bmod 6), \lambda \equiv 0 \quad(\bmod 6)
\end{gathered}
$$

$\mathbb{C}_{6.6 .3}$

$$
P=\{1,2,3,4,5,6\} \text { and } \mathcal{L}=\{123,124,135,236,456,456\}
$$

$$
v \equiv 0 \quad(\bmod 6), \lambda \equiv 0 \quad(\bmod 6)
$$

$\mathbb{C}_{6.6 .4}$

$$
v \equiv 0 \quad(\bmod 6), \lambda \equiv 0 \quad(\bmod 6)
$$

$\mathbb{C}_{6.6 .5}$

$$
P=\{1,2,3,4,5,6\} \text { and } \mathcal{L}=\{123,124,134,256,356,456\}
$$

$$
P=\{1,2,3,4,5,6\} \text { and } \mathcal{L}=\{123,124,135,246,356,456\}
$$

$$
v \equiv 0 \quad(\bmod 6), \lambda \equiv 0 \quad(\bmod 6)
$$

$\mathbb{C}_{6.6 .6}$

$$
P=\{1,2,3,4,5,6\} \text { and } \mathcal{L}=\{123,124,135,346,256,456\}
$$

$$
v \equiv 0 \quad(\bmod 6), \lambda \equiv 0 \quad(\bmod 6)
$$

$\mathbb{C}_{6.6 .7}$

$$
P=\{1,2,3,4,5,6\} \text { and } \mathcal{L}=\{123,124,156,256,345,346\}
$$

$$
v \equiv 0 \quad(\bmod 6), \lambda \equiv 0 \quad(\bmod 6)
$$

$\mathbb{C}_{6.9 .1}$

$$
\begin{aligned}
& \quad P=\{1,2,3,4,5,6,7,8,9\} \text { and } \mathcal{L}=\{123,123,456,456,789,789\} \\
& v \equiv 0(\bmod 9), \lambda \equiv 0(\bmod 4) \text { and } \\
& v \equiv 9(\bmod 18), \lambda \equiv 2(\bmod 4)
\end{aligned}
$$


THE ELECTRONiC JOURNAL OF COMBINATORICS 7 (2000), \#R2

$\mathbb{C}_{6.9 .2}$

$P=\{1,2,3,4,5,6,7,8,9\}$ and $\mathcal{L}=\{123,123,456,457,689,789\}$

$v \equiv 9 s(\bmod 36) \lambda \equiv 0(\bmod 4), s=0,2$;

$\lambda \equiv 0(\bmod 2), s=3 ; \lambda \geq 2, s=1$

$\mathbb{C}_{6.9 .3}$

$$
P=\{1,2,3,4,5,6,7,8,9\} \text { and } \mathcal{L}=\{123,124,356,457,689,789\}
$$

$v \equiv 9 s(\bmod 36) \lambda \equiv 0(\bmod 4), s=0,2$;

$\lambda \equiv 0(\bmod 2), s=3 ; \lambda \geq 2, s=1$

$\mathbb{C}_{6.9 .4}$

$$
P=\{1,2,3,4,5,6,7,8,9\} \text { and } \mathcal{L}=\{123,124,367,489,567,589\}
$$

$v \equiv 9 s(\bmod 36) \lambda \equiv 0(\bmod 4), s=0,2$;

$\lambda \equiv 0(\bmod 2), s=3 ; \lambda \geq 2, s=1$

$\mathbb{C}_{6.9 .5}$

$$
P=\{1,2,3,4,5,6,7,8,9\} \text { and } \mathcal{L}=\{123,124,367,489,568,579\}
$$

$v \equiv 9 s(\bmod 36) \quad \lambda \equiv 0(\bmod 4), s=0,2$;

$\lambda \equiv 0(\bmod 2), s=3 ; \lambda \geq 2, s=1$

$\mathbb{C}_{6.9 .6}$

$$
P=\{1,2,3,4,5,6,7,8,9\} \text { and } \mathcal{L}=\{123,145,246,379,578,689\}
$$

$v \equiv 9 s(\bmod 36) \quad \lambda \equiv 0(\bmod 4), s=0,2$;

$\lambda \equiv 0(\bmod 2), s=3$; any $\lambda, s=1$

$\mathbb{C}_{6.9 .7}$

$$
P=\{1,2,3,4,5,6,7,8,9\} \text { and } \mathcal{L}=\{123,145,267,367,489,589\}
$$

$v \equiv 9 s(\bmod 36) \quad \lambda \equiv 0(\bmod 4), s=0,2$;

$\lambda \equiv 0(\bmod 2), s=3 ; \lambda \geq 2, s=1$

$\mathbb{C}_{6.9 .8}$

$$
P=\{1,2,3,4,5,6,7,8,9\} \text { and } \mathcal{L}=\{123,145,267,389,468,579\}
$$

$v \equiv 9 s(\bmod 36) \lambda \equiv 0(\bmod 4), s=0,2$;

$\lambda \equiv 0(\bmod 2), s=3 ;$ any $\lambda, s=1$ 
THE ELECTROniC JOURNAL OF COMBinatorics 7 (2000), \#R2

$\mathbb{C}_{6.9 .9}$

$$
P=\{1,2,3,4,5,6,7,8,9\} \text { and } \mathcal{L}=\{123,123,456,478,579,689\}
$$

$v \equiv 9 s(\bmod 36) \quad \lambda \equiv 0(\bmod 4), s=0,2$;

$\lambda \equiv 0(\bmod 2), s=3 ; \lambda \geq 2, s=1$

$\mathbb{C}_{6.18 .1}$

$$
\begin{gathered}
P=\{a, b, c, d, e, f, g, h, i, j, k, l, m, n, o, p, q, r\} \text { and } \\
\mathcal{L}=\{a b c, d e f, g h i, j k l, m n o, p q r\} \\
v \equiv 0(\bmod 18), \lambda \equiv 0(\bmod 2)
\end{gathered}
$$

\subsection{Necessary and Sufficient conditions for all $l \leq 4$ and some $l=5,6$}

Theorem 3.1 The necessary conditions for the following $\mathbb{C}$-resolvable designs to exist are sufficient with the addition of $v \neq 6, v \neq 6$ and $\lambda \equiv 2(\bmod 4), v \neq 6$ and $\lambda \equiv 6(\bmod 12)$ to those marked respectively with a "*”, “**", "***":

\begin{tabular}{|c|c|c|c|c|c|}
\hline Configuration & Note & Configuration & Note & Configuration & Note \\
\hline $\mathcal{B}_{1.3 .1}{ }^{*}$ & 1 & $\mathcal{B}_{2.3 .1}{ }^{*}$ & 3 & $\mathcal{B}_{2.6 .1}{ }^{* *}$ & 2,3 \\
\hline $\mathcal{B}_{3.3 .1}{ }^{*}$ & 1 & $\mathcal{B}_{3.9 .1}$ & 2 & $\mathcal{B}_{4.3 .1}{ }^{*}$ & 2 \\
\hline $\mathcal{B}_{4.4 .1}$ & 2 & $\mathcal{B}_{4.6 .1}$ & 2,3 & $\mathcal{B}_{4.12 .1}$ & 1 \\
\hline $\mathcal{B}_{5.3 .1}{ }^{*}$ & 3 & $\mathcal{B}_{5.15 .1}$ & 1 & $\mathcal{B}_{6.3 .1}{ }^{*}$ & 3 \\
\hline $\mathcal{B}_{6.6 .1}{ }^{* * *}$ & 2,3 & $\mathcal{B}_{6.9 .1}$ & 2,3 & $\mathcal{B}_{6.18 .1}$ & 1 \\
\hline
\end{tabular}

Proof: The desired $\mathbb{C}$-resolvable design is equivalent to the existence of a RBIBD whose number of blocks is a multiple of the number of blocks in the former and whose $\lambda$ is a divisor of the former because

1. A parallel class of the RBIBD can be partitioned to form a $\mathbb{C}$-resolvable parallel class.

2. Some multiple of each of the RBIBD can be partitioned into copies of $\mathbb{C}$.

3. A $\mathbb{C}$ parallel class is just an RBIBD parallel class with each block repeated $\mu$ times.

The "Note" indicates which reason(s) should be used for the given configuration.

Theorem 3.2 The necessary conditions for the existence of a $\mathcal{B}_{4.6 .2}$ and a $\mathcal{B}_{4.6 .3}$ are sufficient except possibly if $v=12$. 
Proof: It is well-known that a $3-R G D D(3, n)$ (or a Kirkman triple system of order $3 n)$ exists if and only if $n \equiv 1(\bmod 2)$ and also that a $3-R G D D_{2}(3, n)$ exists for all integers $n \neq 2$. We use the master by ingredient construction using for a master a $3-R G D D(3, n)$ if $v \equiv 6(\bmod 12)$ and a $3-R G D D_{2}(3, n)$ if $v \equiv 0(\bmod 12), v \geq 24$. For auto-ingredient use example 2.2 (taken 4 times in the first case and 2 times in the second one) for the Pasch and example 2.3 (taken twice in the first case and 1 time in the second one) for the FIFA.

In order to fill in groups we need $\mathbb{C}_{4.6 .2}$ and .3 resolvable designs:

$\mathcal{B}_{4.6 .2}, V=Z_{5} \cup\{\infty\}, \lambda=4$. The $5 \mathbb{C}$-parallel classes are:

$\{\{\infty, 1+i, 3+i\},\{\infty, 2+i, 4+i\},\{0+i, 1+i, 2+i\}$, $\{0+i, 3+i, 4+i\} \bmod 5\}, i \in Z_{5}$

$\mathcal{B}_{4.6 .3}, V=Z_{5} \cup\{\infty\}, \lambda=4$. The $5 \mathbb{C}$-parallel classes are

$\{\{\infty, 0+i, 1+i\},\{\infty, 2+i, 4+i\},\{0+i, 1+i, 4+i\}$, $\{0+i, 2+i, 4+i\} \bmod 5\}, i \in Z_{5}$

Theorem 3.3 If there is a $\operatorname{RBIBD}(v, 5, \mu)$ then there is a $\mathcal{B}_{5.5 . x}$ for the following values of $x$ and lambda: $x=1$ and $\lambda \equiv 0(\bmod 6 \mu) ; x=2$ and $\lambda \equiv 0(\bmod 3 \mu)$.

Proof: The existence of a $\operatorname{RBIBD}(v, 5, \mu)$ is known in many cases, see [6] for a survey of the results. The proof follows from the existence of a $\mathcal{B}_{5.5 . x}$, and using one parallel class of blocks as the groups to create the master RGDD needed. $x=1$ and $\lambda=6, x=2$ and $\lambda=3$.

$\mathcal{B}_{6.5 .1}, V=Z_{5}, \lambda=6$. The $4 \mathbb{C}$-parallel classes are:

$\{032,032,014,214,314\}$

$\{012,012,034,134,234\}$,

$\{123,123,104,204,304\}$,

$\{013,013,024,124,324\}$.

$\mathcal{B}_{6.5 .2}, V=Z_{5}, \lambda=3$. The $2 \mathbb{C}$-parallel classes are:

$\{032,034,021,341,241\}$,

$\{041,042,013,423,123\}$. 


\subsection{Further sufficient conditions for $l=6$}

In this ,section we examine some sufficient conditions which fall short of the necessary conditions. In each case there is a range of uncertainty which further woek may narrow.

Theorem 3.4 For each $v \equiv 6(\bmod 12), \lambda \equiv 0(\bmod 6)$ and $v \equiv 0(\bmod 12), v \geq 24, \lambda \equiv 0(\bmod 12)$, there exists a $\mathcal{B}_{6.6 .3}$.

Proof: We proceed as in Theorem 3.2 by using the same master, the following 6-auto ingredient configuration and $\mathcal{B}_{6.6 .3}$ with $v=6, \lambda=6$.

A 6-auto ingredient configuration $\mathbb{C}_{6.6 .3}$ :

$V=Z_{6}$. The groups are: $\{0,3\},\{1,4\},\{2,5\}$. The $4 \mathbb{C}$-parallel classes are:

$$
\begin{aligned}
& \{012,015,024,123,534,534\}, \\
& \{312,315,324,120,045,045\}, \\
& \{315,312,354,150,024,024\}, \\
& \{015,012,054,153,234,234\} .
\end{aligned}
$$

$\mathcal{B}_{\text {6.6.3 }}, V=Z_{5} \cup\{\infty\}, \lambda=6$. The $5 \mathbb{C}$-parallel classes are: $\{\{\infty, 0+i, 1+i\},\{\infty, 0+i, 2+i\},\{\infty, 1+i, 4+i\}$,$$
\{0+i, 1+i, 3+i\},\{2+i, 3+i, 4+i\} \text {, }
$$$$
\{2+i, 3+i, 4+i\} \bmod 5\}, i \in Z_{5}
$$

Theorem 3.5 For each $v \equiv 0(\bmod 6), \lambda \equiv 0(\bmod 12), v \neq 24$, there exists a $\mathcal{B}_{6.6 .2}$ and $a \mathcal{B}_{6.6 .5}$.

Proof: A 6-auto ingredient configuration $\mathbb{C}_{6.6 .2}$ :

$V=Z_{6}$. The groups are: $\{0,3\},\{1,4\},\{2,5\}$. The $4 \mathbb{C}$-parallel classes are:

$$
\begin{aligned}
& \{012,012,024,135,435,435\}, \\
& \{312,312,324,105,405,405\}, \\
& \{015,015,054,123,423,423\}, \\
& \{315,315,354,102,402,402\} .
\end{aligned}
$$


$\mathcal{B}_{6.6 .2}, V=Z_{5} \cup\{\infty\}, \lambda=12$. The $10 \mathbb{C}$-parallel classes are:

$\{\{\infty, 0+i, 1+i\},\{\infty, 0+i, 1+i\},\{\infty, 1+i, 3+i\}$,

$\{0+i, 2+i, 4+i\},\{2+i, 3+i, 4+i\}$,

$\{2+i, 3+i, 4+i\} \bmod 5\}, i \in Z_{5}$

$\{\{\infty, 0+i, 2+i\},\{\infty, 0+i, 2+i\},\{\infty, 2+i, 3+i\}$,

$\{0+i, 1+i, 4+i\},\{1+i, 3+i, 4+i\}$,

$\{1+i, 3+i, 4+i\} \bmod 5\}, i \in Z_{5}$

A 6-auto ingredient configuration $\mathbb{C}_{6.6 .5}$ :

$V=Z_{6}$. The groups are: $\{0,3\},\{1,4\},\{2,5\}$. The $4 \mathbb{C}$-parallel classes are:

$$
\begin{aligned}
& \{012,015,024,153,243,543\}, \\
& \{045,042,051,423,513,213\}, \\
& \{342,345,321,450,210,510\}, \\
& \{042,045,021,453,213,513\} .
\end{aligned}
$$

$\mathcal{B}_{6.6 .5}, V=Z_{5} \cup\{\infty\}, \lambda=12$ The $10 \mathbb{C}$-parallel classes are:

$\{\{\infty, 0+i, 1+i\},\{\infty, 0+i, 2+i\},\{\infty, 1+i, 3+i\}$,

$\{0+i, 2+i, 4+i\},\{1+i, 3+i, 4+i\}$,

$\{2+i, 3+i, 4+i\} \bmod 5\}, i \in Z_{5}$

$\{\{\infty, 0+i, 4+i\},\{\infty, 0+i, 2+i\},\{\infty, 4+i, 3+i\}$,

$\{0+i, 2+i, 1+i\},\{4+i, 3+i, 1+i\}$,

$\{2+i, 3+i, 1+i\} \bmod 5\}, i \in Z_{5}$

Theorem 3.6 Let $\lambda$ be even. The necessary conditions for a $\mathbb{C}$-resolvable $\mathcal{B}_{6.9 . x}$, $x=2,3,4,8$, to exist are sufficient with the addition of $v \neq 18$.

Proof: A 2-auto ingredient configuration $\mathbb{C}_{6.9 .2}$ :

$V=Z_{9}$. The groups are: $\{1,3,0\},\{2,5,7\},\{4,6,8\}$. The $3 \mathbb{C}$-parallel classes are:

$$
\begin{aligned}
& \{176,176,432,435,280,580\}, \\
& \{378,378,415,412,560,260\}, \\
& \{470,470,182,185,236,536\} .
\end{aligned}
$$

$\mathcal{B}_{6.9 .2}, V=Z_{9}, \lambda=2$. The $4 \mathbb{C}$-parallel classes are:

$\{123,123,705,708,564,864\}$,

$\{247,247,180,186,053,653\}$,

$\{258,258,673,671,340,140\}$,

$\{260,260,387,384,715,415\}$. 
A 2-auto ingredient configuration $\mathbb{C}_{6.9 .3}$ :

$V=Z_{9}$. The groups are: $\{0,1,3\},\{2,5,7\},\{4,6,8\}$. The $3 \mathbb{C}$-parallel classes are:

$$
\begin{aligned}
& \{124,128,470,873,563,560\}, \\
& \{541,543,176,378,620,820\}, \\
& \{580,581,047,176,423,623\} .
\end{aligned}
$$

$\mathcal{B}_{6.9 .3}, V=Z_{9}, \lambda=2$. The $4 \mathbb{C}$-parallel classes are:

$$
\begin{aligned}
& \{130,134,085,486,627,527\}, \\
& \{120,124,056,457,638,738\}, \\
& \{178,176,802,604,453,253\}, \\
& \{704,703,428,326,851,651\} .
\end{aligned}
$$

A 2-auto ingredient configuration $\mathbb{C}_{6.9 .4}$ :

$V=Z_{9}$. The groups are: $\{1,2,3\},\{4,6,8\},\{0,5,7\}$. The $3 \mathbb{C}$-parallel classes are:

$$
\begin{aligned}
& \{145,147,526,738,026,038\}, \\
& \{167,160,728,034,528,534\}, \\
& \{365,367,518,724,018,024\} .
\end{aligned}
$$

$\mathcal{B}_{6.9 .4}, V=Z_{9}, \lambda=2$. The $4 \mathbb{C}$-parallel classes are:

$$
\begin{aligned}
& \{374,378,124,125,608,605\}, \\
& \{167,163,287,280,453,450\}, \\
& \{301,302,481,486,752,756\}, \\
& \{701,704,851,853,623,264\} .
\end{aligned}
$$

A 2-auto ingredient configuration $\mathbb{C}_{6.9 .8}$ :

$V=Z_{9}$. The groups are: $\{1,2,3\},\{4,5,6\},\{0,7,8\}$. The $3 \mathbb{C}$-parallel classes are:

$$
\begin{aligned}
& \{147,160,428,735,638,025\}, \\
& \{160,158,627,034,537,824\}, \\
& \{158,147,520,836,430,726\} .
\end{aligned}
$$

For a $\mathcal{B}_{6.9 .8}$ with $\lambda=2$ take two copies of the following Kirkman triple system of order 9:

$\mathcal{B}_{6.9 .8}, V=Z_{9}, \lambda=1$. The $2 \mathbb{C}$-parallel classes are:

$$
\begin{aligned}
& \{023,067,245,318,658,741\}, \\
& \{162,150,287,634,537,048\} .
\end{aligned}
$$


THE ELECTRONiC JOURNAL OF COMBinatorics 7 (2000), \#R2

\section{Conclusions}

$\mathcal{B}_{6.6 .4}, V=Z_{5} \cup\{\infty\}, \lambda=12$. The $10 \mathbb{C}$-parallel classes are:

$\{\{\infty, 0+i, 1+i\},\{\infty, 0+i, 2+i\},\{\infty, 1+i, 2+i\}$,

$\{0+i, 3+i, 4+i\},\{1+i, 3+i, 4+i\}$,

$\{2+i, 3+i, 4+i\} \bmod 5\}, i \in Z_{5}$

$\{\{\infty, 0+i, 3+i\},\{\infty, 0+i, 2+i\},\{\infty, 3+i, 2+i\}$,

$\{0+i, 1+i, 4+i\},\{3+i, 1+i, 4+i\}$,

$\{2+i, 1+i, 4+i\} \bmod 5\}, i \in Z_{5}$

$\mathcal{B}_{6.6 .6}, V=Z_{5} \cup\{\infty\}, \lambda=6$. The $5 \mathbb{C}$-parallel classes are:

$\{\{\infty, 0+i, 1+i\},\{\infty, 0+i, 4+i\},\{\infty, 1+i, 3+i\}$,

$\{1+i, 4+i, 2+i\},\{0+i, 3+i, 2+i\}$,

$\{2+i, 3+i, 4+i\} \bmod 5\}, i \in Z_{5}$

$\mathcal{B}_{6.6 .7}, V=Z_{5} \cup\{\infty\}, \lambda=12$. The $10 \mathbb{C}$-parallel classes are:

$\{\{\infty, 0+i, 1+i\},\{\infty, 0+i, 3+i\},\{\infty, 4+i, 2+i\}$,

$\{0+i, 2+i, 4+i\},\{1+i, 3+i, 2+i\}$,

$\{1+i, 3+i, 4+i\} \bmod 5\}, i \in Z_{5}$

$\{\{\infty, 0+i, 1+i\},\{\infty, 1+i, 4+i\},\{\infty, 3+i, 2+i\}$,

$\{1+i, 2+i, 3+i\},\{0+i, 2+i, 4+i\}$,

$\{0+i, 3+i, 4+i\} \bmod 5\}, i \in Z_{5}$

$\mathcal{B}_{6.9 .2}, V=Z_{9}, \lambda=3$. The $6 \mathbb{C}$-parallel classes are:

$\{123,123,470,478,650,658\}$,

$\{268,268,174,170,354,350\}$,

$\{348,348,157,152,607,602\}$,

$\{108,108,452,456,372,376\}$,

$\{146,146,278,275,308,305\}$,

$\{240,240,361,367,581,587\}$.

$\mathcal{B}_{6.9 .9}, V=Z_{9}, \lambda=2$. The $4 \mathbb{C}$-parallel classes are:

$\{123,123,465,478,570,680\}$,

$\{158,158,246,270,347,360\}$,

$\{140,140,256,287,357,368\}$,

$\{167,167,245,280,348,350\}$. 
Definition 4.1 A configuration $\mathbb{C}=(P, \mathcal{L})$ is strongly 3-colorable if and only the vertices of $P$ can be colored such that each $l \in \mathcal{L}$ receives one vertex of each color.[2]

Definition 4.2 A coloring of a configuration $\mathbb{C}=(P, \mathcal{L})$ is equitable if and only if all color classes have the same size.[3]

Definition 4.3 A regular configuration is uniform if it has a strong equitable coloring.

For example $\mathbb{C}_{6.9 .2}$ and $\mathbb{C}_{6.9 .9}$ is uniform, $\mathbb{C}_{5.5 .1}$ and $\mathbb{C}_{5.5 .2}$ is not uniform.

Let $\mathbb{C}$ be a uniform configuration, and let $(\mathrm{V}, \mathcal{B})$ be a $\mathbb{C}$-resolvable $\lambda$-TS. We will suppose that the blocks of $\mathcal{B}$ are colored by inheriting the colors of $\mathbb{C}$. Further we will always write the blocks as $\left\{a_{1}, a_{2}, a_{3}\right\}$ where the color of $a_{i}=i$.

Theorem 4.1 Let $\mathbb{C}$ be uniform configuration. Suppose there exist: a $\mathbb{C}$-resolvable $\lambda-T S(v),(V, \mathcal{B}) ; a \mathbb{C}$-resolvable $\lambda-T S(w)$; two orthogonal quasigroups of order $w$, $\left(Z_{w}, \cdot\right)$ and $\left(Z_{w}, \circ\right)$. Then there is a $\mathbb{C}$-resolvable $\lambda-T S(v w)$.

Proof: For each $\alpha \in Z_{w}$ let $T_{\alpha}=\left\{(i, j, i \circ j) \mid i, j \in Z_{w} i \cdot j=\alpha\right\}$ be a transversal of $\left(Z_{w}, \circ\right)$. Let $\mathrm{W}=\left(\mathrm{V} \times Z_{w}\right) \cup T$ and construct a $\mathbb{C}$-resolvable $\lambda$-TS(vw), $(\mathrm{W}, \mathcal{D})$ in the following way:

For each $\mathbb{C}$-parallel class $\mathcal{B}_{x}$ of $(\mathrm{V}, \mathcal{B})$, construct the following $\mathrm{w} \quad \mathbb{C}$-parallel classes of $(\mathrm{W}, \mathcal{D})$,

$$
\mathcal{B}_{x}^{\alpha}=\left\{\left\{a_{i}, b_{j}, c_{i \circ j}\right\} \mid\{a, b, c\} \in \mathcal{B}_{x} \text { and }(i, j, i \circ j) \in T_{\alpha}\right\} .
$$

For each $a \in \mathrm{V}$ let $\left(a \times Z_{w}, \mathcal{E}_{a}\right)$ be a $\mathbb{C}$-resolvable $\lambda$-TS(w). Clearly $\cup_{a \in V} \mathcal{E}_{a}$ is a $\mathbb{C}$-parallel class of $(\mathrm{W}, \mathcal{D})$.

Corollary 4.1 For each $v=9^{n}$ there is a $\mathcal{B}_{6,9,2}$ with $\lambda=3$ and a $\mathcal{B}_{6,9,9}$ with $\lambda=2$.

Proof: The proof follows from Theorem 4.1 and the existence of above $\mathcal{B}_{6,9,2}$ with $v=9, \lambda=3$ and $\mathcal{B}_{6,9,9}$ with $v=9, \lambda=2$.

\section{References}

[1] A.E. Brouwer, "Steiner triple systems without forbidden subconfigurations", Mathematisch Centrum Amsterdam, ZW 104-77, 1977. 
[2] Colbourn, Charles J.; Jungnickel, Dieter(D-GSSN); Rosa, Alexander" The strong chromatic number of partial triple systems". Discrete Appl. Math. 20 (1988), no. $1,31-38$.

[3] Colbourn, Charles; Haddad, Lucien; Linek, Vclav "Equitable embeddings of Steiner triple systems". J. Combin. Theory Ser. A 73 (1996), no. 2, 229-247.

[4] C.J. Colbourn and A. Rosa, Triple Systems, Oxford University Press1999.

[5] Chee, Y. M., Colbourn, C. J. and Ling, A. C. H., "Weakly union-free twofold triple systems, " Annals of Combinatorics , Springer-Verlag 11997.13

[6] Colbourn, C. J. and Dinitz, J. H., ed., CRC Handbook of Combinatorial Designs, CRC Press, New York, 1996.

[7] Dembowski, Peter Finite geometries. Reprint of the 1968 original. Classics in Mathematics. Springer-Verlag, Berlin, 1997.

[8] J.H. Dinitz and D.R. Stinson, Contemporary Design Theory - A Collection of Surveys, John Wiley \& Sons, New York, 1992.

[9] M. J. Granell, T. S. Griggs and E. Mendelsohn, "A small basis for four line configurations in Steiner triple systems", J. Comb. Des. 3 (1995), 51-59.

[10] T.S. Griggs, M.J. de Resmini, and Rosa, A., " Decomposing Steiner triple systems into four-line configurations", Ann. Discrete Math. 52 (1992), 215-226.

[11] T.S. Griggs, E. Mendelsohn, and A. Rosa, "Simultaneous decompositions of Steiner triple systems", Ars Combinat. 37 (1994), 157-173.

[12] Griggs T., Murphy, J. and Phelan, J.S., "Anti-Pasch Steiner triple systems", J. Combin. Inf. Syst. Sci. 15 (1990), 79-84.

[13] T.S. Griggs, and A. Rosa, "Avoidance in triple systems" Acta Math. Univ. Comen. 63 (1994), 117-131.

[14] Hall, M. Jr., Combinatorial Theory, Blaisdell, Toronto, 1967.

[15] Lefmann, H., Phelps, K.T. and Rödl, V., " Extremal problems for triple systems", J. Combin. Des. 1 (1993), 379-394.

[16] Mendelsohn, Eric; Rosa, Alexander " Ubiquitous configurations in Steiner triple systems". J. Combin. Des. 5 (1997), no. 1, 13-31.

[17] Robinson, R. M., " The structure of certain triple systems" Math. Comput. 20 (1975), 223-241. 
[18] Stinson, D. R. and Wei, R., " Some results on quadrilaterals in Steiner triple systems", Discrete Math. 105 (1992), 207-219.

[19] Wallis, W. D., Combinatorial Designs, Dekker 118, New York, 1988. 